\title{
MiR-15a is underexpressed and inhibits the cell cycle by targeting CCNE1 in breast cancer
}

\author{
QIFENG LUO ${ }^{1}$, XIAOYU LI ${ }^{1}$, JIA LI $^{2}$, XIANGJIE KONG ${ }^{1}$, JUNFENG ZHANG ${ }^{1}$, \\ LEI CHEN ${ }^{1}$, YIXIANG HUANG ${ }^{1}$ and LIN FANG $^{1}$ \\ ${ }^{1}$ Department of General Surgery, Shanghai Tenth People's Hospital, Tongji University School of Medicine, Shanghai 200072, \\ P.R. China; ${ }^{2}$ Department of Microbiology and Genetic Institute, Paris-Sud 11 University, Paris, France
}

Received May 24, 2013; Accepted July 5, 2013

DOI: $10.3892 /$ ijo.2013.2034

\begin{abstract}
MicroRNAs (miRNAs) are a small class of noncoding RNAs that are widely dysregulated in various cancers. They act as either oncogenes or tumor suppressor genes in human cancer. The purpose of this study was to examine the expression levels of miR-15a in human breast cancer and its potential role in disease pathogenesis. The expression levels of miR-15a were measured by quantitative polymerase chain reaction (qPCR) in 40 breast cancer specimens and adjacent normal breast tissues. MTT assays, colony formation assays, transwell chamber migration assays, cell cycle and apoptosis assays were used to explore the potential function of miR-15a in MDA-MB-231 human breast cancer cells. Luciferase reporter assays were performed to validate the regulation of a putative target of miR-15a, in corroboration with qPCR and western blot assays. We found that the expression of miR-15a was lower in breast cancer specimens compared with adjacent normal tissues $(\mathrm{p}<0.05)$. Overexpression of miR-15a inhibited cellular growth, suppressed migration and arrested cells at the G1 phase, but did not promote cellular apoptosis. Luciferase assays indicated that miR-15a can bind with its putative target site in the 3'-untranslated region (3'-UTR) of CCNE1, suggesting that CCNE1 is a direct target of miR-15a. qPCR and western blot analysis indicated that the overexpression of miR-15a results in the downregulation of CCNE1 at the mRNA and protein levels. Taken together, the upregulation of miR-15a expression causes cellular growth inhibition, suppression of migration and G1 phase arrest by targeting CCNE1. These findings suggest that miR-15a may act as a tumor suppressor gene in breast cancer and that, in the future, it could be used as a therapeutic target for the treatment of breast cancer.
\end{abstract}

Correspondence to: Dr Lin Fang, Department of General Surgery, Shanghai Tenth People's Hospital, Tongji University School of Medicine, 301 Middle Yanchang Road, Zhabei District, Shanghai 200072, P.R. China

E-mail: fangzhuren301@163.com

Key words: miR-15a, CCNE1, cell cycle, breast cancer

\section{Introduction}

MicroRNAs (miRNAs) are composed of approximately 20-25 nucleotide-non-coding RNAs that may silence post-transcriptional protein expression through two axes: first, by binding to complementary target messenger RNAs to target them for degradation; and second, inhibiting mRNA translation into proteins (1). There has been significant evidence showing that miRNAs regulate as many as $30 \%$ of the human protein coding genes (2). Moreover, they can function as oncogenes or tumor suppressor genes by altering regulation of their targets in many cancers $(3,4)$. Indeed, studies have shown that miRNAs are involved in a variety of processes including tumor cell proliferation, differentiation, and apoptosis. Moreover, their differing expressions can lead to different human cancers $(5,6)$. Subsequently, miRNA research has become a hot spot in breast cancer research whereby miRNAs are believed to have broad prospects in terms of diagnosis and treatment of this disease (7-10)

Recent studies indicate that miR-15a is downregulated in chronic lymphocytic leukemia (11), prostate cancer (12), osteosarcoma (13), keratocystic odontogenic tumors (14) and breast cancer (15). Furthermore, through overexpression of miR-15a, curcumin can reduce the expression of Bcl-2 and subsequently induce apoptosis in MCF-7 breast cancer cells (15). However, the mechanism by which miR-15a contributes to breast cancer tumorigenesis is still unclear.

Cyclin E1 (CCNE1), one member of the cyclin E family, can associate with and activate cyclin-dependent kinase 2 (CDK2). CCNE1 is a positive regulator of G1/S phase transition and is essential for cell cycle re-entry from G0 phase. In many human tumors, CCNE1 is overexpressed and the level of both protein and kinase activity is often deregulated relative to the cell cycle, as demonstrated in human breast epithelial cells (16). One study has indicated that deregulation of CCNE1 is an early event in the development of breast cancer (17). Furthermore, overexpression of CCNE1 in patients with breast cancer is associated with worse prognosis $(18,19)$. CCNE1 can be repressed by some miRNAs, for example, a study has demonstrated the involvement of miR-16 in its regulation in human endothelial progenitor cells (20).

In this study, we first demonstrated that miR-15a expression is significantly lower in breast cancer specimens when 
compared with that of adjacent normal tissues. Its overexpression inhibited proliferation of MDA-MB-231 breast cancer cells, in association with inhibition of migration and disruption of the cell cycle by targeting CCNE1. These results indicate that miR-15a functions as a tumor suppressor gene, whose dysregulation may be involved in the development of human breast cancer.

\section{Materials and methods}

Specimens. In this study, 40 paired breast cancer specimens and adjacent normal breast tissues were collected from the Department of General Surgery of the Shanghai Tenth People's Hospital. These samples were immediately snap-frozen in liquid nitrogen. All samples were confirmed as invasive, ductal breast cancer by trained pathologists. No patients received chemotherapy or radiotherapy prior to surgery.

Cell lines and transfection. The MDA-MB-231 breast cancer cells and HEK293T cells used in this study were purchased from the ATCC (Manassas, VA, USA). Cells were grown in Dulbecco's modified Eagle's medium (DMEM; Gibco, USA) supplemented with $10 \%$ fetal bovine serum (FBS; Gibco), penicillin $(100 \mathrm{U} / \mathrm{ml})$ and streptomycin $(100 \mu \mathrm{g} / \mathrm{ml})$ (Enpromise, China). Cells were incubated at $37^{\circ} \mathrm{C}$ in a humidified chamber supplemented with $5 \% \mathrm{CO}_{2}$.

For transfections, cells $\left(2 \times 10^{5}\right)$ were added into each well of a 6-well plate and cultured with DMEM medium without either serum or antibiotics. When the density of MDA-MB-231 breast cancer cells reached 30-40\%, miR-15a mimics (GenePharma Co., Ltd., Shanghai, China) and Lipofectamine transfection reagent (Invitrogen, USA) were each diluted in $500 \mu \mathrm{l}$ DMEM medium, at a ratio of $1 \mu \mathrm{g}: 3 \mu \mathrm{l}$ and incubated for $5 \mathrm{~min}$ at room temperature (RT). The two mixtures were then gently combined and incubated for a further 20-30 min at RT. Subsequently, 1,000 $\mu \mathrm{l}$ of the complexes were added to each well. After 5-6 h of incubation, DMEM medium was replaced by DMEM with $10 \%$ FBS. Cells were incubated at $37^{\circ} \mathrm{C}$ in a $\mathrm{CO}_{2}$ incubator for $48 \mathrm{~h}$ prior to further testing.

Quantitative reverse-transcription polymerase chain reaction ( $q R T-P C R)$. MicroRNAs were harvested according to the instructions of the miRcute miRNA isolation kit (Tiangen, Beijing, China). For miRNA qPCR, the miR-15a primer, U6 primer and EzOmics SYBR qPCR kit were purchased from Biomics Biotechnology Inc. (Jiangsu, China). The amplification procedure was as follows: $94^{\circ} \mathrm{C}$ for $10 \mathrm{~min}$, followed by 40 cycles at $94^{\circ} \mathrm{C}$ for $20 \mathrm{sec}, 61^{\circ} \mathrm{C}$ for $30 \mathrm{sec}$ and $72^{\circ} \mathrm{C}$ for $30 \mathrm{sec}$.

For quantification of CCNE1 mRNA expression, total RNA was isolated using TRIzol (Invitrogen) and cDNA was generated by reverse transcription using the PrimeScript RT-PCR kit in accordance with the manufacturer's instructions (Takara). Real-time PCR was performed on a 7900HT fast RT-PCR instrument using SYBR-Green and the following primers: CCNE1: 5'-TTTCAGGGTATCAGTGGTG-3' (sense), and 5'-ACATGGCTTTCTTTGCTC-3' (antisense); GAPDH: 5'-AAGGTCGGAGTCAACGGATT-3' (sense), and 5'-CTGGA AGATGGTGATGGGATT-3' (antisense). The PCR parameters for relative quantification were as follows: $5 \mathrm{~min}$ at $94^{\circ} \mathrm{C}$, followed by 30 cycles of $30 \mathrm{sec}$ at $94^{\circ} \mathrm{C}, 45 \mathrm{sec}$ at $57^{\circ} \mathrm{C}$ and $45 \mathrm{sec}$ at $72^{\circ} \mathrm{C}$. Each sample was tested in triplicate. The relative expression was calculated following the relative quantification equation $=2^{-\Delta \Delta \mathrm{CT}}(21)$.

Cell proliferation assay. Cell proliferation was assessed using an MTT assay kit (Sigma, Santa Clara, CA, USA) in accordance with the manufacturer's instructions. Briefly, $\sim 4-5 \mathrm{~h}$ after transfection of miR-15a mimics, cells administered either 50 or $100 \mathrm{nmol} / 1 \mathrm{miR}-15 \mathrm{a}$ mimics or negative control (NC) were trypsinized and counted. Cells from each condition were plated (3,000/well) in 96-well plates (BD Biosciences, USA) and incubated at $37^{\circ} \mathrm{C}$ in a humidified chamber supplemented with $5 \% \mathrm{CO}_{2}$. Cell proliferation was assessed at 24, 48, 72 and $96 \mathrm{~h}$. The optical density (OD) of each well was measured with a microplate spectrophotometer at $490 \mathrm{~nm}$. All experiments were performed in biological triplicate.

Colony formation assay. After transfection with $100 \mathrm{nmol} / \mathrm{l}$ miR-15a or NC, cells were trypsinized, counted, and seeded for colony formation assay in 6-well plates at 300/well. During colony growth, the culture medium was replaced every 3 days. On the 8th day after seeding, the cells were fixed and then stained with crystal violet, and the number of colonies was counted. The colony was counted only if it contained $>50$ cells. Each treatment was carried out in triplicate.

Transwell chamber migration assay. The transwell migration assay was performed in a 24-well transwell chamber system. The filter was washed with the serum-free DMEM, and placed between the lower and upper chambers. The lower chambers contained DMEM with $10 \%$ FBS. The miR-15a or NC transfected MDA-MB-231 cells were trypsinized, resuspended in DMEM with $0.1 \%$ BSA, transferred to the upper chambers, and incubated at $37^{\circ} \mathrm{C}$ in $5 \% \mathrm{CO}_{2}$. After $20 \mathrm{~h}$, the filter was removed, the upper surface of the filter containing non-migrating cells was cleared using a wet cotton swab, and the cells remaining on the underside were stained with crystal violet. Five fields of each well were randomly gated and counted. Then, glacial acetic acid was used to dissolve crystal violet and the OD was measured at $573 \mathrm{~nm}$. Each treatment was carried out in triplicate.

Cell cycle and apoptosis assay. Thirty-six hours after transfection with the miR-15a mimics, or NC, cells were trypsinized and centrifuged at 1,000 rpm for $5 \mathrm{~min}$, followed by two washes in cold PBS. Then, $3.0 \mathrm{ml}$ ice-cold ethanol was added in a dropwise fashion and cells were allowed to fix for $\geq 30 \mathrm{~min}$. A total of $250 \mu \mathrm{l} 0.05 \mathrm{~g} / \mathrm{l}$ propidium iodide (PI) staining solution was added into each sample and incubated for $30 \mathrm{~min}$ at RT. Cells were then analyzed on a flow cytometer (FACSCanto $^{\mathrm{TM}}$ II, BD Biosciences).

For Annexin V staining, miR-15a and NC groups of adherent cells were harvested and incubated with Annexin V incubation reagent (prepared by combining $10 \mu \mathrm{l} 10 \mathrm{X}$ binding buffer, $10 \mu \mathrm{l}$ PI, $1 \mu \mathrm{l}$ Annexin V-FITC and $79 \mu 1$ deionized, distilled $\mathrm{H}_{2} \mathrm{O}$ ) at a ratio of $10^{5}-10^{6}$ cells $/ 100 \mu \mathrm{l}$ for $15 \mathrm{~min}$ at RT in the dark. All samples were processed by flow cytometry (FACSCanto ${ }^{\mathrm{TM}}$ II, BD Biosciences). FACS analyses were performed at least three times with reproducible results. 
Luciferase assay. We used a total PCR reaction volume of $50 \mu 1$ to amplify the 3'-UTR of CCNE1 containing the predicted miR-15a binding site using the Primer star kit (Takara), in accordance with the manufacturer's instructions. The primers used were: 5'-ATTCTAGGCGATCGCTCGAGC CACCCCATCCTTCTCCA-3' (sense); 5'-TTTATTGCGGCC AGCGGCCGCTCAAAAACAGTATTATCTTTATTAAA-3' (antisense). Fragments were then subcloned into the XhoI site in the 3'-UTR of firefly luciferase of the psiCHECK-2 reporter vector. psiCHECK-2/CCNE1 3'-UTR reporter plasmids (100 ng) were co-transfected with the miR-15a mimics or NC (100 nmol/l) into HEK293T cells, at 70\% confluence, using Lipofectamine 2000 (Invitrogen), according to the manufacturer's instructions. After $30 \mathrm{~h}$, cells were lysed and reporter activity was assessed using the Dual-luciferase reporter assay system (Promega, USA) in accordance with the manufacturer's protocol. Firefly luciferase activity was normalized to renilla luciferase activity.

Western blot analysis. The protein expression levels were detected by western blotting. Whole cell protein extracts [lysis buffer: $50 \mathrm{mM}$ Tris- $\mathrm{HCl}$ (pH 7.5), $150 \mathrm{mM} \mathrm{NaCl}, 1 \% \mathrm{NP} 40$, $1 \mathrm{mM}$ phenylmethylsulfonyl fluoride, and $19 \mathrm{mM}$ protease inhibitor cocktail (Sigma-Aldrich, USA)] were quantified by bicinchoninic acid assay (Pierce, USA). Protein samples were separated by $10 \%$ sodium dodecyl sulfate polyacrylamide gel electrophoresis and transferred onto nitrocellulose membranes (Beyotime, China). Immune complexes were formed by incubation of membranes with primary antibody (Epitomics, USA) overnight at $4^{\circ} \mathrm{C}$. Blots were washed and incubated for $1 \mathrm{~h}$ with horseradish peroxidase-conjugated anti-rabbit secondary antibody. Immunoreactive protein bands were detected using an Odyssey Scanning system.

Statistical analysis. Data are presented as the mean \pm standard error of mean from at least three independent experiments. The two-tailed t-test was used to draw a comparison between groups. The null hypothesis was rejected at the 0.05 level.

\section{Results}

Expression of miR-15a is decreased in human breast cancer. To investigate the expression level of miR-15a in breast cancer, we analyzed levels of miR-15a in 40 paired invasive ductal breast cancer specimens and associated normal adjacent tissues by qRT-PCR. As depicted in Fig. 1, the $2^{-\Delta \Delta C t}$ value of miR-15a was significantly decreased in breast cancer tissues (2.125 \pm 0.096$)$ compared with that of normal adjacent tissues $(9.570 \pm 0.337)(\mathrm{p}<0.05)$.

Suppression of breast cancer cell proliferation by miR-15a. To explore the potential impact of miR-15a on the proliferation of breast cancer cells, miR-15a mimics were used and viability was measured by the MTT assay in MDA-MB-231. Compared with the NC group, miR-15a significantly repressed the growth of breast cancer cells. Suppression of cell growth by miR-15a was time- and dose-dependent, whereby miR-15a at a concentration of $100 \mathrm{nmol} / \mathrm{l}$ at $72 \mathrm{~h}$ showed the greatest inhibitory effect $(p<0.05)$ (Fig. 2). Proliferation was also assessed by colony formation assay (Fig. 3). We found that the number of

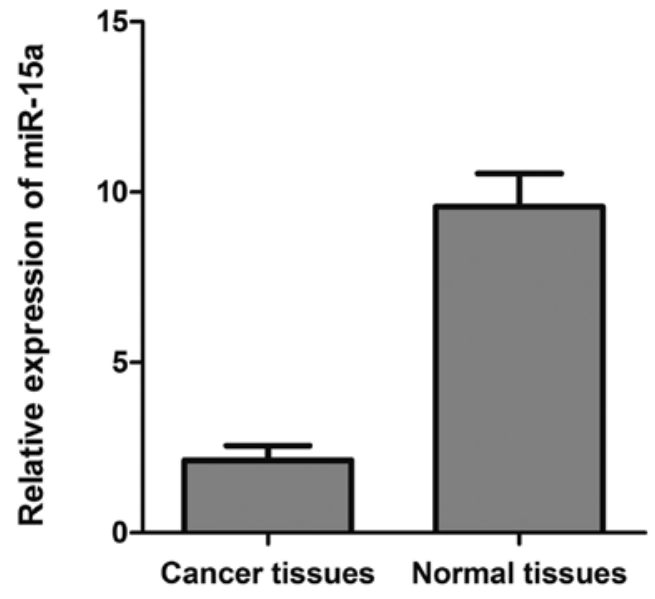

Figure 1. miR-15a is significantly decreased in breast cancer tissues. The mRNA expression of miR-15a was measured by qRT-PCR in breast cancer tissues and adjacent normal breast tissue. Graph represents the $2^{-\Delta \Delta C t}$ values \pm SEM, ${ }^{*}$ p $<0.05$.

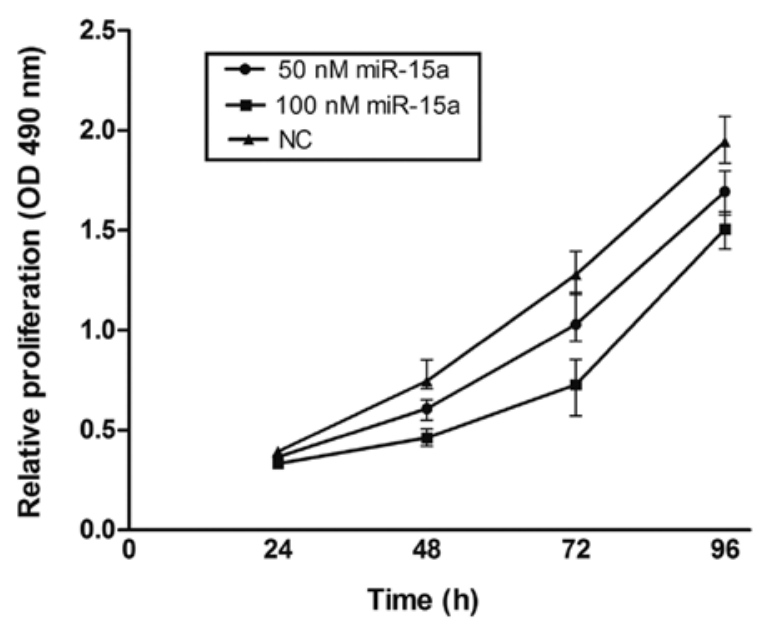

Figure 2. miR-15a inhibits cell proliferation. The MTT assay was performed to monitor the proliferation level of MDA-MB-231 breast cancer cells after transfection with control (NC) or miR-15a mimics at the indicated concentrations. The optical density of each well was measured at the indicated time-points at $490 \mathrm{~nm}$ with a microplate spectrophotometer. Graph represents OD $490 \mathrm{~nm} \pm$ SEM, ${ }^{*} \mathrm{p}<0.05$.

colonies of the miR-15a group was $56.25 \pm 4.151$, which was significantly less than that of the NC group $(100.8 \pm 2.175)$ $(\mathrm{p}<0.05)$. Thus, these data suggest that miR-15a significantly suppresses the proliferation of MDA-MB-231 breast cancer cells.

miR-15a inhibits migration of MDA-MB-231 cells. The transwell migration assay is a useful method to investigate migratory ability. Our results showed that $20 \mathrm{~h}$ after transfection the number of migrating cells in the miR-15a group was less than that in the NC group. Furthermore, the OD $573 \mathrm{~nm}$ values, derived by solubilization of crystal violet staining, revealed a significant decreased from $0.497 \pm 0.009$ to $0.229 \pm 0.010(\mathrm{p}<0.05)$ in the NC and miR-15a groups, respectively. These data indicate that the migratory ability of MDA-MB-231 cells might be inhibited by miR-15a (Fig. 4). 

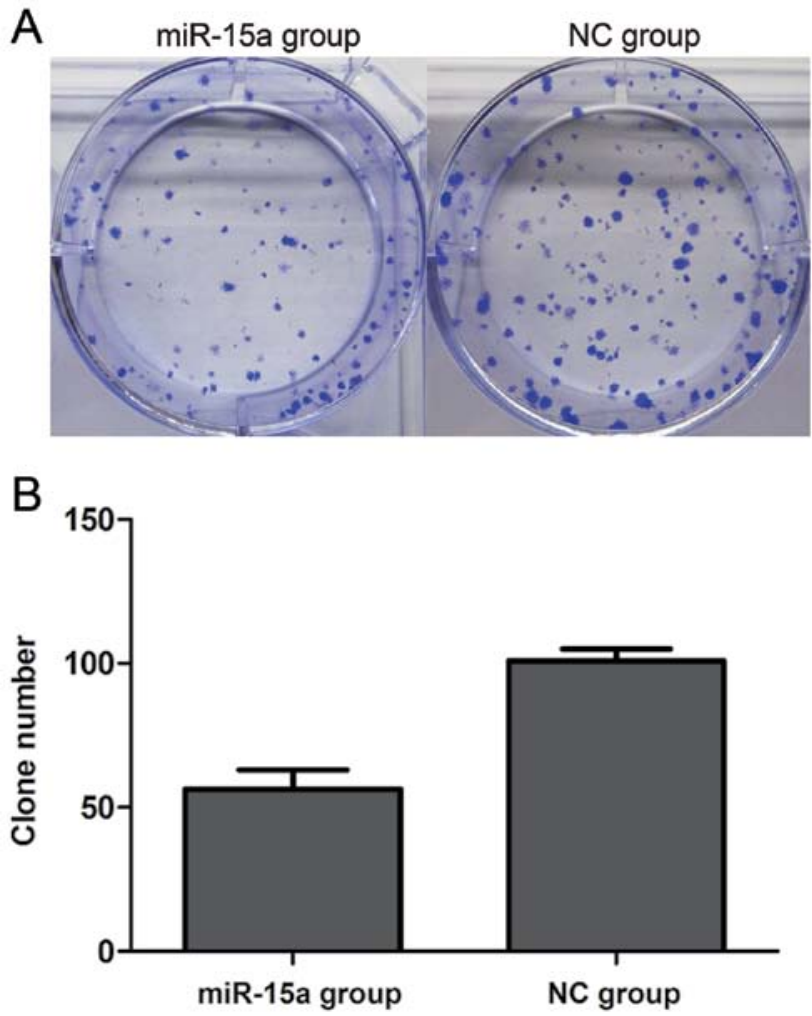

Figure 3. Colony formation assay. (A) Representative images of crystal violet stained colonies in MDA-MB-231 cells transfected with miR-15a or NC. (B) Quantification of the clone numbers, ${ }^{*} \mathrm{p}<0.05$.

miR-15a disrupts the cell cycle of MDA-MB-231 cells. Thirty-six hours after the transfection of miR-15a mimics $(100 \mathrm{nmol} / \mathrm{l})$, flow cytometry analysis revealed that the percentage of G0/G1 phase cells $(54.88 \pm 0.175 \%)$ dramatically increased in the miR-15a group, when compared with that of the NC group $(46.16 \pm 0.182 \%)(\mathrm{p}<0.05)$, while the proportion of $\mathrm{S}$-phase cells decreased in the miR-15a group $(31.30 \pm 0.116 \%)$ compared with that of the NC group $(36.62 \pm 0.205 \%)(\mathrm{p}<0.05)$. The percentage of $\mathrm{G} 2 / \mathrm{M}$ phase cells also decreased in the miR-15a group (13.71 $\pm 0.229 \%)$ compared with that of the NC group $(17.23 \pm 0.076 \%)(p<0.05)$. These findings suggest that miR-15a can initiate G0/G1 phase arrest and that upregulation of miR-15a expression could lead to the reduction of S-phase and G2/M phase cells (Fig. 5). However, our data indicate that there was no difference in apoptosis between the miR-15a and $\mathrm{NC}$ groups (Fig. 6).

MiR-15a regulates CCNE1 expression by targeting CCNE1 in $M D A-M B-231$ cells. To validate the possibility that miR-15a may target CCNE1 in breast cancer cells we first searched for putative targets using the miRanda, targetscan and miRBase databases. We found a potential binding site for miR-15a in the 3'-UTR of CCNE1 mRNA, which was located 247-254 bp downstream from the 5'-end of the CCNE1 3'-UTR. We then cloned the putative binding site into a luciferase reporter construct and used it to measure the effects of miR-15a mimics in MDA-MB-231 cells. We found that luciferase activity was significantly lower in cells co-transfected with psiCHECK-2/ CCNE1 3'-UTR and miR-15a, when compared with that of co-transfection with NC (Fig. 7) $(\mathrm{p}<0.05)$. Thus the results of
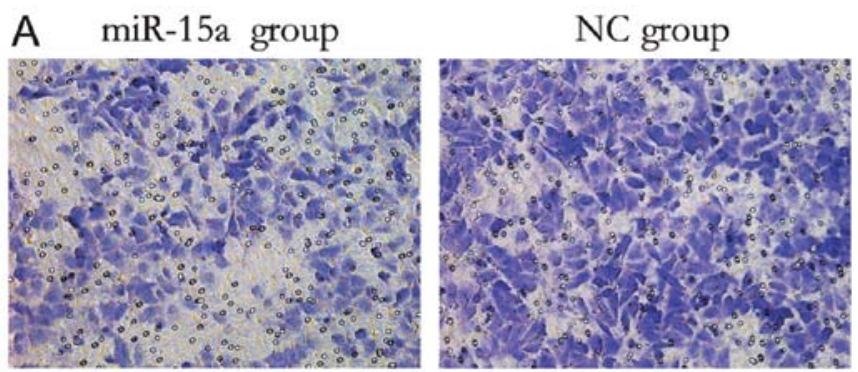

B

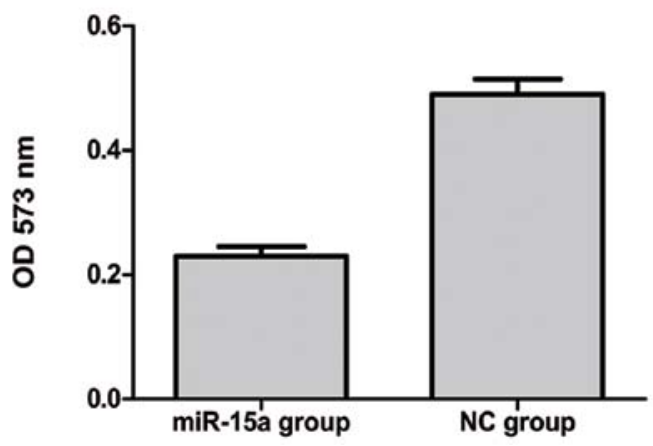

Figure 4. miR-15a inhibits the migration of MDA-MB-231 breast cancer cells. Cell migration ability was analyzed by the Transwell chamber assay $20 \mathrm{~h}$ after miR-15a, or NC transfection. (A) Representative images of crystal violet stained MDA-MB-231 migratory cells transfected with miR-15a or NC. (B) Quantification of the migratory cells by solubilization of crystal violet and spectrophotometric reading at OD 573. Data represent mean \pm SEM, " $\mathrm{p}<0.05$.

this experiment show that miR-15a could directly interact with the CCNE1 3'-UTR fragments of the psiCHECK-2 reporter plasmid, which could lead to the degradation of renal luciferase mRNA. Moreover, we performed qPCR and western blot analysis. QPCR indicated that CCNE1 expression was significantly lower in the miR-15a mimics group compared with that of the NC group, with relative expression levels of $1.957 \pm 0.050$ and $5.335 \pm 0.169(\mathrm{p}<0.05)$, respectively (Fig. 8A). In western blot analysis, CCNE1 protein expression was also significantly decreased by the overexpression of miR-15a (Fig. 8B).

\section{Discussion}

Breast cancer is one of the most common malignant tumors in women. Besides surgery, chemotherapy is the major therapeutic method. However, there are still patients that exhibit resistance to chemotherapy, as demonstrated through early recurrence and metastasis, leading to poor prognosis. This is especially true for triple-negative breast cancers (22), which do not express estrogen receptor, progesterone receptor or the human epidermal growth factor receptor-2. In this case, it is particularly important to explore new treatments. Currently, the search for novel therapeutic agents for breast cancer is one of the hot topics of breast cancer research (23-25).

In this study, we examined the expression level of miR-15a in human breast cancer and its potential role in disease pathogenesis. First, we detected the expression level of miR-15a in human breast cancer specimens by qRT-PCR. The results showed that miR-15a was significantly lower in breast cancer tissues than in normal breast tissues. Similar findings have 

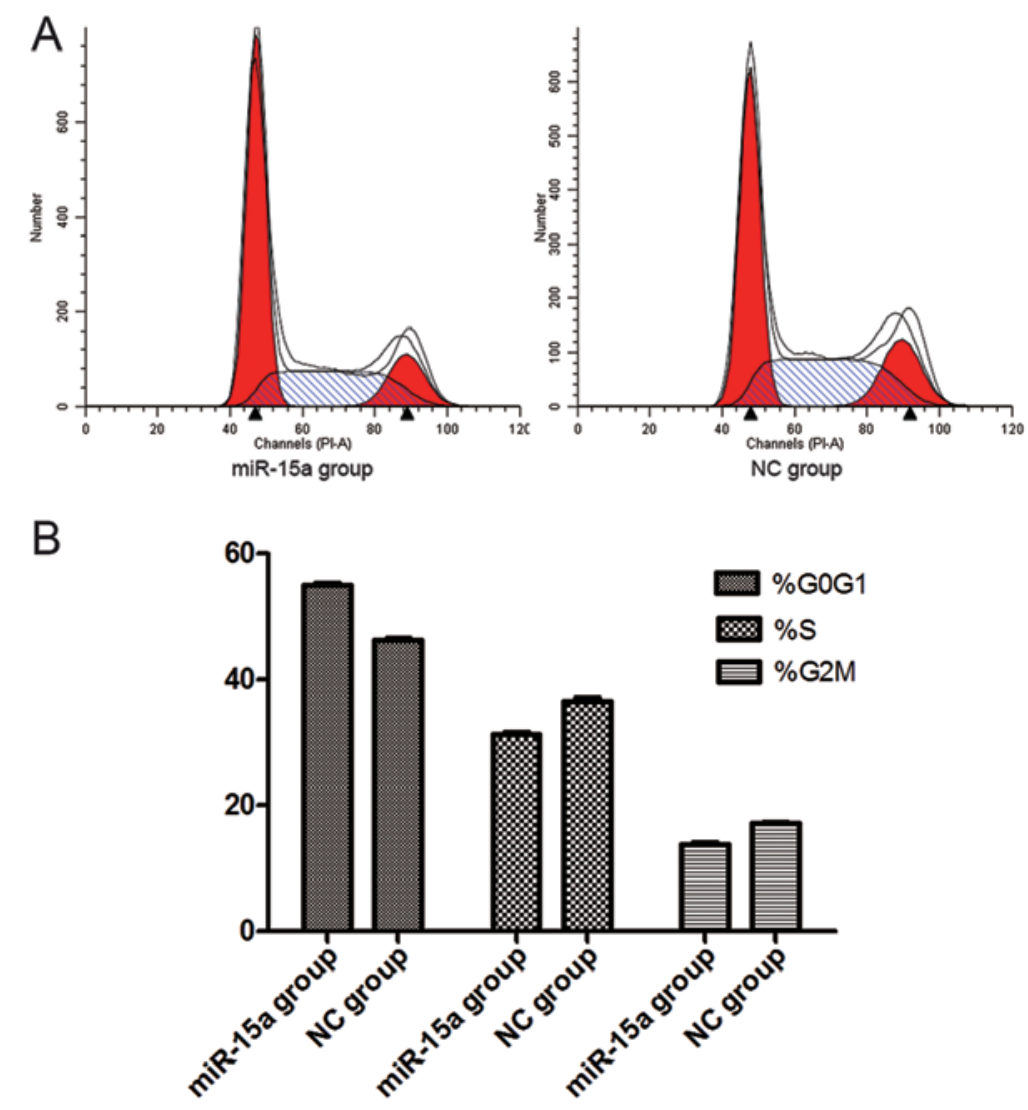

Figure 5. Cell cycle distribution. (A) Cell cycle distribution was analyzed by flow cytometry $36 \mathrm{~h}$ after transfection of MDA-MB-231 breast cancer cells with $100 \mathrm{nmol} / 1 \mathrm{miR}-15 \mathrm{a}$ mimics or NC. (B) The respective proportion of G0/G1 phase, S-phase and G2/M phase of miR-15a and NC groups, "p $<0.05$.

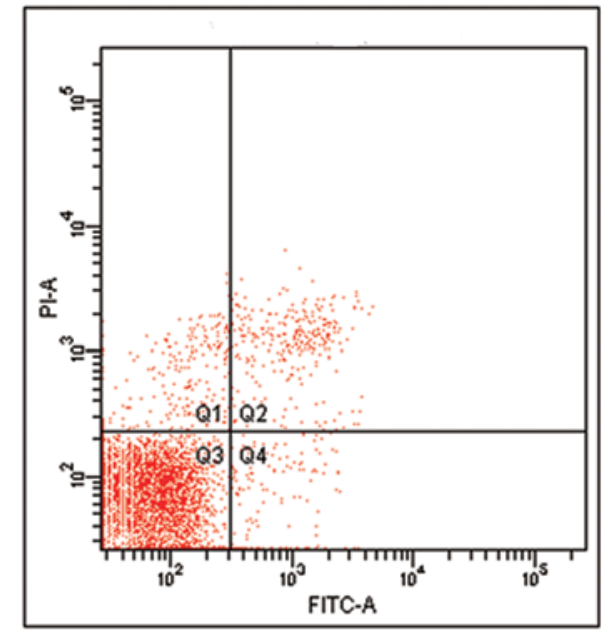

miR-15a group

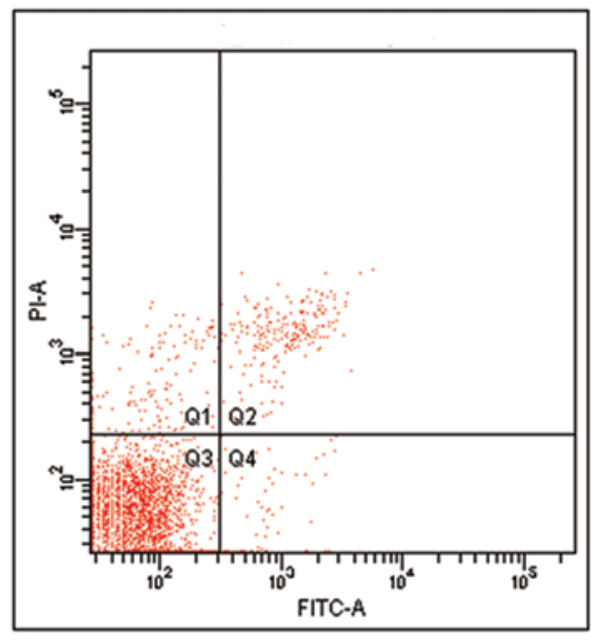

NC group

Figure 6. Apoptosis analysis of cells transfected with miR-15a. Cells transfected with miR-15a or NC were stained with PI and analyzed by flow cytometry.

been reported in other cancer types $(11,12)$, which indicates that downregulation of miR-15a is common in human cancer specimens and cell lines. Next, we transfected miR-15a mimics into MDA-MB-231 cells to generate its overexpression. This led to significant inhibition of cellular proliferation as measured by MTT, as well as a reduction in the colony number as determined by clone formation assay. These two experiments indicate that miR-15a represses the growth of breast cancer cells. Using the transwell migration assay, we found that the overexpression of miR-15a in breast cancer cells could suppress their migratory ability. We found that miR-15a distinctly arrests cancer cells at the G1 phase when compared with the cell cycle of NC groups. However, our study found no significant difference in apoptosis between the 
A

3`-GUGUUUGGUAUACACGACGAU-5`has-miR-15a

5`....AgugcGugcucccGaugCugCUA....-3`CCNE1 3`-UTR

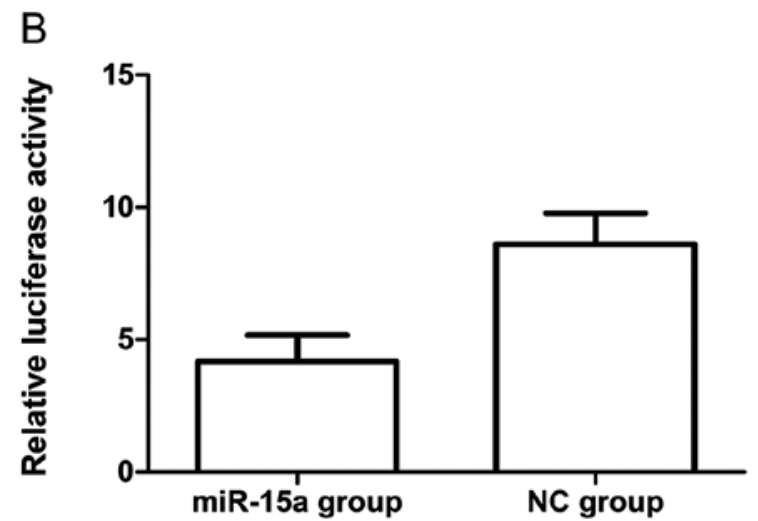

Figure 7. CCNE1 is a direct target of miR-15a. (A) The binding site for miR-15a in the 3'-UTR of CCNE1 mRNA. (B) The relative luciferase activity (renilla/ firefly) was measured in MDA-MB-231 cells after co-transfection of the CCNE1 luciferase construct with either miR-15a or NC, ${ }^{*} \mathrm{p}<0.05$.

A

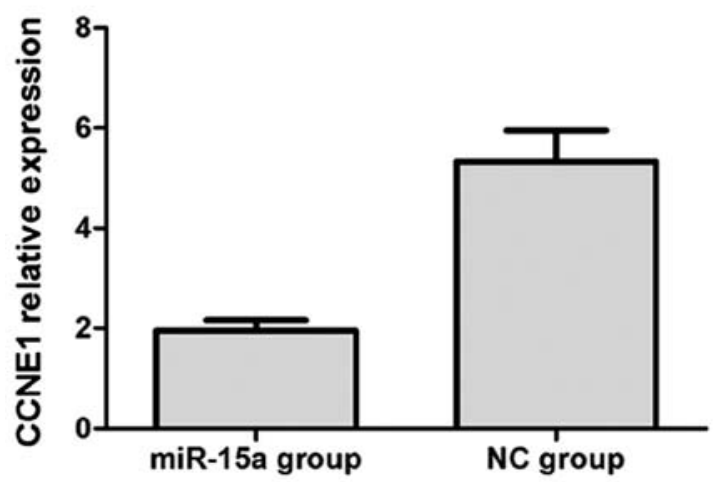

B

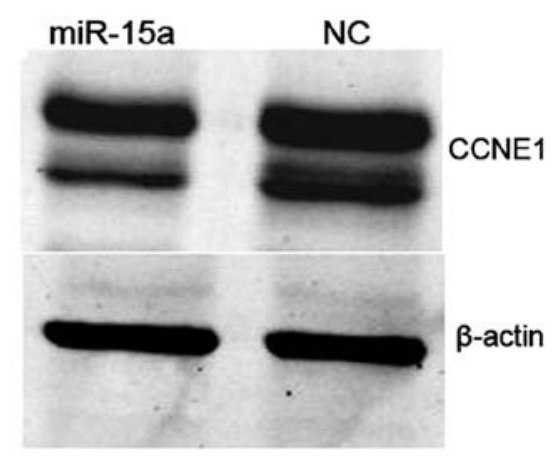

Figure 8. miR-15a inhibits CNNE1 expression. qRT-PCR (A) and western blot (B) analysis of CCNE1 mRNA and protein levels, respectively. $\beta$-actin was used as a control for loading the western blots (B).

miR-15a and NC groups. The vitality of cancer cells is very strong, thus we speculate that this is one possible reason why miRNA-15a could not promote apoptosis.

To investigate the downstream targets of miR-15a that may play a role in mediating its cell function, we searched for putative targets using the miRanda, targetscan and miRBase databases. Through luciferase assays, we predicated CCNE1 as a direct target of miR-15a in MDA-MB-231 cells. Additionally, we found that both the mRNA and protein levels of CCNE1 were significantly lower in miR-15a than those in $\mathrm{NC}$ groups. These findings support the prediction that CCNE1 is a downstream target of miR-15a.

Collectively, our findings suggest that miR15a can disrupt the cell cycle by targeting CCNE1 in MDA-MB-231 cells. We show that its overexpression can reduce cell proliferation and inhibit the migratory ability of cancer cells. Thus, it may be concluded that miR-15a acts as a tumor suppressor gene in breast cancer. Moreover, the luciferase, qPCR and western blot assays illustrate CCNE1 as a downstream target of miR-15a.
The artificial upregulation of miR-15a using CCNE1 as a therapeutic agent could offer a promising new direction for future breast cancer treatment.

\section{Acknowledgements}

This study was made possible with financial support from the National Natural Sciences Foundation of China, for the project 81272240, and the Shanghai Science Committee Foundation (to Lin Fang) (no. STCSM 10411964700). We sincerely thank all the teachers at the Central Laboratory of the Shanghai Tenth People's Hospital for their help and support.

\section{References}

1. Bartel DP: MicroRNAs: genomics, biogenesis, mechanism, and function. Cell 116: 281-297, 2004.

2. Lewis BP, Burge CB and Bartel DP: Conserved seed pairing, often flanked by adenosines, indicates that thousands of human genes are microRNA targets. Cell 120: 15-20, 2005. 
3. Zhang B, Pan X, Cobb GP and Anderson TA: microRNAs as oncogenes and tumor suppressors. Dev Biol 302: 1-12, 2007.

4. Chen C-Z: MicroRNAs as oncogenes and tumor suppressors. N Engl J Med 353: 1768-1771, 2005.

5. Noguchi S, Mori T, Hoshino Y, et al: MicroRNA-143 functions as a tumor suppressor in human bladder cancer T24 cells. Cancer Lett 307: 211-220, 2011.

6. Pallante P, Visone R, Ferracin M, et al: MicroRNA deregulation in human thyroid papillary carcinomas. Endocr Relat Cancer 13: 497-508, 2006

7. Iorio MV, Ferracin M, Liu CG, et al: MicroRNA gene expression deregulation in human breast cancer. Cancer Res 65: 7065-7070, 2005.

8. Ma L, Teruya-Feldstein $\mathrm{J}$ and Weinberg RA: Tumour invasion and metastasis initiated by microRNA-10b in breast cancer. Nature 449: 682-688, 2007.

9. Shi W, Gerster K, Alajez NM, et al: MicroRNA-301 mediates proliferation and invasion in human breast cancer. Cancer Res 71: 2926-2937, 2011

10. Tang D, Zhang Q, Zhao S, et al: The expression and clinical significance of microRNA-1258 and heparanase in human breast cancer. Clin Biochem 46: 926-932, 2013.

11. Sampath D, Liu C, Vasan K, et al: Histone deacetylases mediate the silencing of miR-15a, miR-16, and miR-29b in chronic lymphocytic leukemia. Blood 119: 1162-1172, 2012.

12. Musumeci M, Coppola V, Addario A, et al: Control of tumor and microenvironment cross-talk by miR-15a and miR-16 in prostate cancer. Oncogene 30: 4231-4242, 2011.

13. Cai C-K, Zhao G-Y, Tian L-Y, et al: miR-15a and miR-16-1 downregulate CCND1 and induce apoptosis and cell cycle arrest in osteosarcoma. Oncol Rep 28: 1764-1770, 2012.

14. Diniz MG, Gomes CC, de Castro WH, et al: miR-15a/16-1 influences BCL2 expression in keratocystic odontogenic tumors. Cell Oncol 35: 285-291, 2012.

15. Yang J, Cao Y, Sun J and Zhang Y: Curcumin reduces the expression of Bcl-2 by upregulating miR-15a and miR-16 in MCF-7 cells. Med Oncol 27: 1114-1118, 2010.
16. Spruck CH, Won KA and Reed SI: Deregulated cyclin E induces chromosome instability. Nature 401: 297-300, 1999.

17. Shaye A, Sahin A, Hao Q, Hunt K, Keyomarsi K and Bedrosian I: Cyclin E deregulation is an early event in the development of breast cancer. Breast Cancer Res Treat 115: 651-659, 2009.

18. Keyomarsi K, Tucker SL, Buchholz TA, et al: Cyclin E and survival in patients with breast cancer. N Engl J Med 347: $1566-1575,2002$

19. Sgambato A, Camerini A, Collecchi $\mathrm{P}$, et al: Cyclin E correlates with manganese superoxide dismutase expression and predicts survival in early breast cancer patients receiving adjuvant epirubicin-based chemotherapy. Cancer Sci 100: 1026-1033, 2009.

20. Goretti E, Rolland-Turner M, Leonard F, Zhang L, Wagner DR and Devaux Y: MicroRNA-16 affects key functions of human endothelial progenitor cells. J Leukoc Biol 93: 645-655, 2013.

21. Livak KJ and Schmittgen TD: Analysis of relative gene expression data using real-time quantitative PCR and the 2(-Delta Delta C(T)) method. Methods 25: 402-408, 2001.

22. Bryan BB, Schnitt SJ and Collins LC: Ductal carcinoma in situ with basal-like phenotype: a possible precursor to invasive basallike breast cancer. Mod Pathol 19: 617-621, 2006.

23. Ljungberg BJ, Jacobsen J, Rudolfsson SH, Lindh G, Grankvist K and Rasmuson T: Different vascular endothelial growth factor (VEGF), VEGF-receptor 1 and -2 mRNA expression profiles between clear cell and papillary renal cell carcinoma. BJU Int 98: 661-667, 2006.

24. Tryfonopoulos D, Walsh S, Collins DM, et al: Src: a potential target for the treatment of triple-negative breast cancer. Ann Oncol 22: 2234-2240, 2011.

25. Oliveras-Ferraros C, Vazquez-Martin A, Lopez-Bonet E, et al: Growth and molecular interactions of the anti-EGFR antibody cetuximab and the DNA cross-linking agent cisplatin in gefitinib-resistant MDA-MB-468 cells: new prospects in the treatment of triple-negative/basal-like breast cancer. Int J Oncol 33: $1165-1176,2008$ 